\title{
O impacto do trabalho infantil no setor agrícola sobre a saúde
}

\author{
Alexandre Chibebe Nicolella ${ }^{1}$ \\ Ana Lúcia Kassouf ${ }^{2}$ \\ Alexandre Lahóz Mendonça de Barros ${ }^{3}$
}

Resumo: O objetivo desse artigo é verificar se o trabalho infantil no setor agrícola produz impacto negativo sobre a saúde. Para a análise foram utilizadas as PNADs (Pesquisas Nacionais por Amostra de Domicílios) de 1998 e 2003, que trazem suplemento especial sobre saúde, empregando a técnica econométrica de pseudo-painel. Assim, foram consideradas as crianças de 5 a 15 anos em 1998 e de 10 a 20 anos em 2003. Os resultados mostram que, para os indivíduos economicamente ativos, a atividade no setor agrícola e o trabalho de risco no setor agrícola não diferem dos outros setores, ou seja, trabalhar no setor agrícola não impõe um maior desgaste à saúde em relação aos outros setores da economia. Se considerados todos os indivíduos economicamente ativos, os resultados mostram que o trabalho agrícola não afeta a saúde e que o trabalho não-agrícola tem impacto negativo. Dessa forma, acredita-se que a atuação do governo deve ser setorial, visando uma melhoria no acesso ao sistema de saúde, bem como aos medicamentos, e a promoção da educação materna com relação à saúde.

\footnotetext{
${ }^{1}$ Professor Doutor, Fearp/USP. Av. Bandeirantes, n 3900 - Monte Alegre. CEP: 14040-030 - Ribeirão Preto - SP. E-mail: anicolella@fearp.usp.br

2 Professora Titular Esalq/USP. Av. Pádua Dias, n 11. CEP: 13400-970 - Piracicaba - SP. E-mail: alkassou@esalq.usp.br.

${ }^{3}$ Professor FGV/SP. Rua Itapeva, 47412 andar. CEP: 01332-000 - São Paulo - SP. E-mail: almb@fgvsp.br
} 
Palavras-chave: trabalho infantil agrícola, saúde, pseudo-painel.

Classificação JEL: I12, R21, C23

Abstract: The aim of this dissertation is to identify the causal relation between rural child labour and health. The analysis utilized the PNAD, a Brazilian household survey, from 1998 and 2003. The econometric modeling was based on the pseudo-panel approach and was considered the children from 5 to 15 years old in 1998 and from 10 to 20 years old in 2003. The results show that work and work in risky jobs in the agricultural sector do not differ from those impacts of other sectors. It was also presented, for all individuals that work in the agricultural sector does not impact the health capital and work in the non rural sector impact negatively the health capital. So, the government intervention in rural areas should be different from the one implemented on the urban area to mitigate the impact of child labour on health and also, the government intervention should be implemented in parallel with the improvement of the access to the health system, drugs policies, maternal health education program.

Keywords: child labor, health, pseudo-panel.

JEL Classification: I12, R21, C23

\section{Introdução}

O termo trabalho infantil é utilizado rotineiramente na literatura econômica, no entanto, há diversas distinções entre trabalho infantil e exploração de mão-de-obra infantil ${ }^{4}$. Uma diferenciação é que,

\footnotetext{
${ }^{4}$ Segundo Kassouf (2002), em algumas regiões, a infância refere-se à idade cronológica, enquanto em outras se leva em consideração fatores sócio-culturais e, portanto, a legislação que trata do trabalho infantil também varia de um país para outro. Para a OIT (Organização Internacional do Trabalho), crianças são indivíduos com menos de 15 ou 14 anos em alguns países do terceiro mundo (Levison, 2000). No Brasil, crianças são aqueles que possuem menos de 12 anos (Art $2^{\circ}$ do Estatuto da Criança e do Adolescente, Lei 8.069 de 13 de julho de 1990).
} 
atividades como ajudar nos afazeres domésticos, em lojas etc, são consideradas trabalho infantil, mas não exploração da mão-de-obra infantil. A exploração configura-se quando a atividade desempenhada por uma criança ocorre de forma regular, gerando renda para o próprio sustento e da família (Peres et al., 2003). Convencionou-se chamar de trabalho infantil toda atividade de exploração da mão-de-obra infantil.

Apesar da existência de fatores culturais ligados ao trabalho infantil, o principal motivo associado ao trabalho das crianças é a baixa renda per capita familiar. Pais que não possuem a capacidade de arcar com o mínimo necessário para suprir a subsistência da família se vêem obrigados a inserir suas crianças no mercado de trabalho (Basu, 1999). Além desses, fatores como baixa atratividade da escola (Ray, 1999), baixa capacidade de deixar legado e mercado de crédito imperfeito (Baland e Robinson, 1999) podem influenciar a entrada da criança no mercado de trabalho.

Feita a decisão de trabalhar, o tipo de ocupação torna-se importante. Tem-se, portanto, crianças que trabalham em atividades de alto risco e longos períodos e aquelas que trabalham em atividades de baixo risco e por curtos períodos. Essas diferentes intensidades nas atividades exercem impactos distintos no capital humano adquirido pela criança (Cropper, 1977).

O capital humano pode ser dividido em duas partes: a primeira refere-se à educação e a segunda, à saúde (denominado neste texto como capital humano saúde ou capital saúde). Seguindo a idéia proposta por Grossman (1972), a saúde pode ser vista como um estoque de capital durável que produz tempo de vida saudável. Assim, o indivíduo nasce com um estoque inerente que deprecia ao longo da vida e aumenta por meio de investimentos. Além dessas duas formas de capital, há ainda os capitais físico, social e o fisiológico (Fogel, 2004). Dessa forma, há indicações da existência de uma relação de troca entre trabalho e condição de saúde da criança, sendo que essas condições geram conseqüências de longo, médio e curto prazo para o indivíduo e para a nação (Muurinen, 1982; Grossman, 2000; Alsan, Bloom e Canning, 2004; Emerson e Portela, 2003; Strauss, Thomas, 1998; Sohn, 2000). ${ }^{5}$

${ }^{5}$ Curto, médio e longo prazo estão relacionados aos anos de vida do indivíduo, ou seja, tem caráter biológico e não econômico propriamente dito. 
Apesar de a idéia sobre capital humano saúde ser relativamente antiga, as evidências empíricas das conseqüências do trabalho infantil sobre a saúde são escassas.

Um estudo longitudinal (de dezessete anos) conduzido na Índia mostrou que meninos entre 10 e14 anos que receberam salário por seu trabalho possuíam menor crescimento do que aqueles que freqüentavam a escola (Satyanarayanan et al., 1986 apud O'Donnell et al., 2002). Em Gana, Fentimam et al., (2001 apud O’Donnell et al., 2002) encontrou diferenças de morbidade entre os jovens da zona rural que freqüentavam a escola e os que não freqüentavam, argumentando que essa diferença, aparentemente, advém do trabalho infantil de risco. Kassouf et al. (2001) e Guiffrida et al. (2001) determinaram o efeito do trabalho na fase jovem no estado de saúde auto avaliado na fase adulta. Os resultados dos dois estudos mostram que a entrada precoce no mercado de trabalho aumenta a probabilidade de o indivíduo relatar pior estado de saúde. O’Donnell et al., (2002) não encontraram evidências de que o trabalho infantil agrícola provoca impactos na saúde no curto prazo, mas, sim, no longo prazo. Assim, indivíduos que trabalharam na infância apresentaram mais problemas de saúde após cinco anos em relação àqueles que não trabalharam. Observando que os efeitos de curto prazo ou contemporâneos possuem pouca evidência, Rosati e Straub (2004) examinaram o impacto de longo prazo do trabalho infantil na saúde, controlando e não controlando os efeitos não observáveis específicos das famílias. Os dados foram obtidos da Pesquisa Nacional de Condições de Vida da Guatemala e indicaram que o trabalho infantil é danoso para a saúde da criança no longo prazo.

O objetivo deste artigo é verificar se o trabalho infantil no setor agrícola tem impacto negativo sobre a saúde da criança e, caso sim, se esse impacto é distinto em relação aos setores não-agrícolas.

Poucos e recentes são os trabalhos internacionais que tratam o tema com rigor dentro da ótica econômica. No Brasil também há muito pouco trabalho envolvendo esta temática. Assim, esse estudo vem preencher uma lacuna importante no desenvolvimento da discussão científica sobre trabalho infantil no Brasil, principalmente na discussão sobre o trabalho no setor agrícola.

Além dessa introdução, esse trabalho é composto por mais 4 seções. 
Na seção 2 estão descritas as bases de dados utilizadas, algumas características das variáveis e a especificação econométrica (teórica e empírica). Na seção 3 serão elaborados os principais resultados descritivos e econométricos alcançados e, finalmente, a última seção é composta pelas conclusões.

\section{Metodologia}

\subsection{As bases de dados}

Para a análise do impacto do trabalho infantil agrícola na saúde da criança foram utilizadas duas bases de dados, a PNAD 1998 e a PNAD 2003. Essas duas pesquisas trazem um suplemento especial sobre saúde individual.

O suplemento sobre saúde na PNAD ocorreu nos anos de 1981, 1986, 1988, 1998 e 2003. No entanto, as pesquisas não são totalmente compatíveis, visto que, nos anos de 1981 a 1988, as questões abordaram exclusivamente temas relacionados ao acesso aos serviços de saúde e, para o ano de 1986, não há uma pergunta que permite a classificação dos indivíduos quanto ao seu status de saúde. Atualmente, as PNADs de 1998 e 2003 são as pesquisas com o maior detalhamento da caracterização individual da saúde no Brasil.

A pesquisa é feita anualmente, excetuando os anos de censo, realizada no último trimestre do ano. No caso das PNADs 1998 e 2003, o mês de referência foi setembro. Quanto à abrangência, a pesquisa é dividida em cinco grandes regiões sendo elas: Sul, Sudeste, CentroOeste, Nordeste e Norte - esta última refere-se somente a parcela urbana (exceção para o Tocantins). A pesquisa de 1998 totalizou 344.975 pessoas pesquisadas nessas regiões e a de 2003, 384.834 pessoas.

A PNAD é realizada por meio de amostras probabilísticas de domicílios obtidas em três estágios de seleção: unidades primárias (municípios), unidades secundárias (setores censitários) e unidades terciárias (unidades domiciliares). O processo de expansão da amostra utiliza estimadores de razão, no qual a variável independente é a projeção da população residente, segundo tipo de área (região metropolitana e 
não-metropolitana). Para o ano de 1998, essas projeções consideram a evolução populacional ocorrida entre os censos de 1980 e 1991, sob a hipótese de crescimento associado a taxas de fecundidade, mortalidade e migração. Para o ano de 2003, as projeções são feitas utilizando os censos 1991 e 2000.

\subsection{Características das variáveis}

A estimativa do impacto do trabalho infantil na saúde do indivíduo seria relativamente fácil se não houvesse o problema da endogeneidade, composta pela heterogeneidade não observada e pela simultaneidade.

O problema de heterogeneidade não observada é similar ao problema de variáveis omitidas. As medidas de saúde e a atividade laboral são dependentes do estoque de saúde, ou seja, indivíduos com maior estoque de saúde serão mais facilmente empregados e terão medidas de saúde melhores. No entanto, essa característica individual, estoque de saúde, não é de fácil mensuração e, em geral, está ausente dos bancos de dados. Outro fator heterogêneo é a preferência de tempo. Pais futuro-orientados terão maior estímulo em investir na saúde de seus filhos e evitar ao máximo a entrada no mercado de trabalho. De forma oposta, pais presentes-orientados farão menor investimento em saúde e hesitarão menos em colocar seus filhos no mercado de trabalho (Becker e Mulligam, 1997). Portanto, a forma como se desconta as utilidades no tempo é outro fator heterogêneo não observado.

Considera-se, ainda, uma variação positiva, ceteris paribus no estoque de capital saúde no tempo. Essa variação irá aumentar a quantidade de trabalho ofertado e esse aumento no trabalho elevará a depreciação do estoque de saúde. O aumento na depreciação impactará negativamente a acumulação do estoque de capital. Esse impacto negativo, por sua vez, irá afetar o tempo e a produtividade do trabalho no período posterior. Esse processo, em que o estoque de saúde determina parcialmente o trabalho e vice-versa é conhecido como simultaneidade, e representa o segundo componente da endogeneidade. 


\subsection{Especificação econométrica}

\subsubsection{Especificação teórica}

Para contornar o problema exposto na sub-seção 2.2, o modelo que melhor se adequaria à analise do impacto do trabalho infantil agrícola sobre a saúde seria um de efeito fixo com dados em painel. Caso houvesse, no Brasil, uma pesquisa com características de painel, esse modelo poderia ser facilmente elaborado. Esse tipo de pesquisa, contudo, não existe para a análise da relação em questão - trabalho infantil e saúde. Uma possível solução é a utilização da técnica de pseudo-painel, primeiramente proposta por Deaton (1985) e Browning, Deaton e Irish (1985) como uma forma de contornar o problema da falta de dados longitudinais ${ }^{6}$.

A fonte de informação, nesse caso, são cortes seccionais repetidos (CSR), em que não é possível seguir os indivíduos no tempo. É apenas possível seguir grupos ou coortes no tempo. Apesar de os CSR não fornecerem a dinâmica dentro de cada coorte, ou seja, não é possível saber se um indivíduo rico hoje foi pobre no passado, essa técnica é capaz de controlar efeitos fixos não observados (Deaton, 1997).

A limitação dessa alternativa está na impossibilidade da utilização de variáveis defasadas para controle dos efeitos específicos individuais, ou seja, $\operatorname{cov}\left(y_{i t}, y_{i t-1}\right)$ não está disponível (Moffitt, 1993). Como vantagens, essa metodologia não sofre de atrito, como nas pesquisas em painel, que pode ser construída para qualquer característica da distribuição de interesse e não somente a média, permitindo a combinação de bases de dados distintas (Moffitt, 1993, Deaton, 1997 e Menezes-Filho, $2001)^{7}$. Neste artigo, será utilizada a proposta elaborada por Moffitt (1993), na qual considera os dados individuais.

A questão é: quais as restrições que devem ser impostas para estimar modelos consistentes na ausência de $\operatorname{cov}\left(y_{i t}, y_{i t-1}\right)$ ? Começa-se incorporando aos modelos tradicionais a impossibilidade de seguir os indivíduos no tempo. Assim tem-se ${ }^{8}$ :

\footnotetext{
${ }^{6}$ Essa seção está baseada em Moffitt (1993).

${ }^{7} \mathrm{O}$ atrito ocorre quando os indivíduos pesquisados deixam a amostra de forma não aleatória.

${ }^{8}$ Optou-se por começar pela abordagem simplificada para que houvesse maior compreensão da técnica.
} 


$$
\begin{aligned}
& h_{i(t) t}^{*}=\beta x_{i(t) t}+\theta_{i(t)}+u_{i(t) t} \\
& h_{i(t) t}=\left\{\begin{array}{l}
1 \text { se } h_{i(t) t}^{*}>0 \\
0 \text { se } h_{i(t) t}^{*} \leq 0
\end{array}, i(t)=1, \ldots, N, t=1, \ldots, T \text { e } u_{i(t) t} \sim N(0,1) .\right.
\end{aligned}
$$

Sendo $i(t)$ o subscrito que indica que em cada ponto no tempo os indivíduos são distintos, $h_{i(t) t}^{*}$ representa o status de saúde ${ }^{9}, x_{i(t) t}$ é a variável independente, $u_{i(t) t}$ é o erro estocástico, homocedástico e não auto-correlacionado e $\theta_{i(t)}$ são os efeitos específicos individuais não observados, tais como estoque de saúde e preferências, correlacionados com algumas das variáveis independentes do modelo.

Pode se observar que a estimação tradicional da equação acima levaria a $\beta$ inconsistentes, pois $\theta_{i(t)}$ é não observado e não ortogonal a $x_{i(t) t}$. Busca-se, portanto, um estimador de variável instrumental (IV) capaz de produzir estimativas consistentes de $\beta$, sendo que esse instrumento para $x_{i(t) t}$ deve ser assintoticamente não correlacionado com $\theta_{i(t)}$.

Admitindo-se que a amostra advém de uma população fechada ${ }^{10}$, é possível construir grupos baseados em variáveis não dependentes do tempo, sendo a variável mais utilizada a data ou coorte de nascimento. Assim, ao invés de indivíduos, acompanham-se as coortes e os efeitos específicos das coortes ao longo do tempo $\left(c_{i(t)}\right)$. O instrumento para $x_{i(t) t}$ deve ser baseado em uma projeção linear sobre funções de tempo, $t$, e da coorte, $c_{i(t)}$, ou seja:

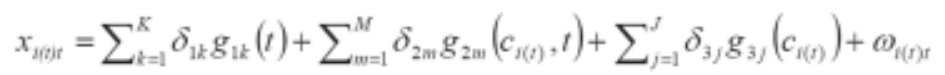

no qual $g_{1 k}$ são funções do tempo, $g_{2 m}$ são funções da interação entre tempo e coorte e $g_{3 j}$ são funções da coorte, sendo essa equação estimada por mínimos quadrados ordinários. Provavelmente, esse instrumento está relacionado $\operatorname{com} \theta_{i(t)}$, gerando um problema de inconsistência do estimador.

Para solucionar tal problema, pode-se decompor os efeitos fixos, $\theta_{i(t)}$,

\footnotetext{
${ }^{9}$ A descrição detalhada das variáveis utilizadas na modelagem será elaborada na próxima seção.

${ }^{10}$ População fechada é aquela no qual não há migração, morte ou nascimento.
} 
na média da amostra da coorte para cada $t$ e mais um desvio da média, $\theta_{t(t)}=\bar{\theta}_{c t}+v_{t(t)}$. Avançando, decompõe-se a média da amostra da coorte em média populacional da coorte, mais um desvio representando o erro da amostragem, $\bar{\theta}_{c t}=\bar{\theta}_{c}{ }^{*}+v_{c(t)}^{\prime}$. Por substituição, tem-se que:

$$
\theta_{i(t)}=\bar{\theta}_{c}^{*}+v_{c(t)}^{\prime}+v_{i(t)}
$$

Assim, $\bar{\theta}_{\epsilon}^{*}$ pode ser representado por binárias na equação princi$\mathrm{pal}^{11}$. A consistência do estimador em dois estágios (2SLS) mais binárias de coortes requer que $N \rightarrow \infty$ enquanto o número de coortes permaneça constante. Dessa forma, $v_{c(t)}^{\prime} \rightarrow 0$ e a distribuição de $v_{i(t)}$ torna-se independente de $t .{ }^{12}$ Portanto, as variações das variáveis independentes no tempo dentro de cada coorte irão representar mudanças comportamentais e não mudanças nas características não observáveis da amostra e, dessa forma, o pseudo-painel terá as mesmas características de um painel verdadeiro (Menezes-Filho, 2001). Generalizando o modelo, tem-se que:

$$
\begin{aligned}
& h_{i(t) t}^{*}=\beta_{1} \tau_{i(t) t}+\beta_{2} \mathbf{X}_{i(t) t}+\theta_{i(t)}+u_{i(t) t} \\
& h_{i(t) t}=\left\{\begin{array}{l}
1 \text { se } h_{i(t) t}^{*}>0 \\
0 \text { se } h_{i(t) t}^{*} \leq 0
\end{array}, i(t)=1, \ldots, N, t=1, \ldots, T \text { e } u_{i(t) t} \sim N(0,1) .\right.
\end{aligned}
$$

sendo $h_{i(t) t}$ o status de saúde da criança no tempo $t$, e $\tau_{i(t) t}$ a variável independente que representa o trabalho no setor agrícola e potencialmente correlacionado com $\theta_{i(t)}$. A projeção linear sobre a qual o método de variável instrumental é construído pode ser assim descrito:

$$
\underset{\substack{i(t) t \\(1 \times 1)}}{\tau_{(1 \times \mathrm{M})(\mathrm{M} \times 1)}^{s}}=\underset{(1 \times \mathrm{L})(\mathrm{L} \times 1)}{\delta_{1}} \boldsymbol{W}_{i(t) t}+\underset{i(t) t}{\delta_{2}} \boldsymbol{Z}_{i(t)}+\omega_{i(t)}
$$

no qual $\delta_{1}$ e $\delta_{2}$ são vetores de coeficientes, $\boldsymbol{W}_{i(t) t}$ o vetor de variáveis tempo-variantes não correlacionadas com $\theta_{i(t)}, \boldsymbol{Z}_{i(t)}$ vetor de variáveis tempo-invariantes (como sexo, raça, local de residência, coorte etc) e o $\omega_{i(t) t}$ vetor de erros. Essa equação é a forma generalizada da eq. (2) e representará a estimativa do primeiro estágio.

Novamente, como instrumentos baseados em efeitos de coorte, provavelmente estão correlacionados $\operatorname{com} \theta_{i(t)}$ a seguinte decomposição do

${ }^{11}$ Como admitiu-se que a população é fechada, então $\bar{\theta}_{c}^{*}$ é constante.

12 2SLS: Two Stage Least Square ou mínimo quadrados em dois estágios. 
efeito fixo é necessária para consistência do estimador:

$$
\theta_{i(t)}=\underset{(1 \times L)}{Z_{i(L \times 1)}^{\prime}} \underset{\substack{(L \times 1) \\ v_{i(t)}}}{\gamma}
$$

sendo $\gamma$ um vetor de coeficientes e $v_{i(t)}$ os efeitos individuais restantes condicionados a $Z_{i(t)}$. Essa equação é a forma generalizada de eq. (3),

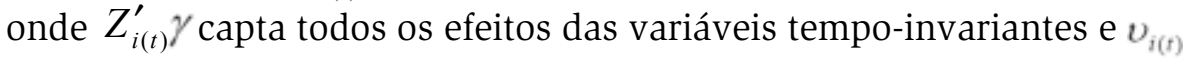
representa a combinação dos erros da eq. (3).

Para que haja consistência, é necessário que plim $\left[\begin{array}{cc}\frac{1}{N T}\left[\begin{array}{ll}\hat{\tau} & Z\end{array}\right]^{\prime} v\end{array}\right]=0$ e que $\left[\begin{array}{ll}\hat{\tau} & Z\end{array}\right]$ seja posto completo $(1+\mathrm{L})$, no qual $\hat{\tau}$ é a variável predita na eq. (5). Essa consistência é atingida quando $N \rightarrow \infty$ sendo $T$ constante. Além disso, se a matriz de variáveis independentes incluir variáveis tempo-invariantes, seus coeficientes não podem ser identificados se essas estiverem correlacionadas com $\theta_{i(t)}$ (Moffitt, 1993).

\subsubsection{Especificação empírica}

A base de dados foi estruturada para que se tenha em 1998 todos os indivíduos de 5 a 15 anos que morem com os pais (nesse artigo consideramos tanto a mãe quanto o pai - chamado de família nuclear). Seguindo esses indivíduos no tempo, em 2003, foram coletados os indivíduos entre 10 a 20 anos com o mesmo tipo de estrutura familiar, ou seja, a família nuclear. Assim os indivíduos com 5 anos em 1998 e os indivíduos com 10 anos em 2003 fazem parte da mesma coorte.

Esse trabalho propõe quatro equações distintas para a análise do trabalho infantil rural e seu impacto na saúde. A primeira seleciona as crianças que trabalham e analisa se o trabalho exercido no setor agrícola tem efeito distinto daquele dos setores não-agrícolas. A segunda equação proposta seleciona somente as crianças que exercem trabalho de risco e analisa se o trabalho agrícola de risco possui impacto distinto em relação ao trabalho de risco de outros setores da economia. A terceira equação seleciona todos os indivíduos que moram na área rural e analisa se o trabalho tem impacto negativo na saúde. A última equação é similar a terceira, mas usa somente os indivíduos que vivem na área urbana. 
O modelo econométrico adotado para todas as equações foi o probit binário com variável independente endógena (Wooldridge, 2002). Assim, a primeira equação que contém todos os indivíduos que moram na área rural poderá ser assim descrita:

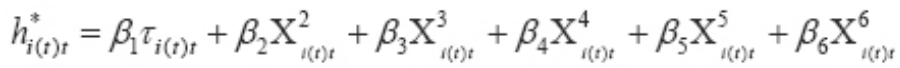

$$
\begin{aligned}
& +\beta_{7} \mathrm{X}_{((t) t}^{7}+\beta_{8} \bar{\theta}_{c}^{*}+u_{i(t) t} \\
& \tau_{i(t) t}=\beta_{9} \mathrm{X}_{i(t) t}^{8}+\beta_{10} \mathrm{X}_{i(t) t}^{9}+\beta_{11} \mathrm{X}_{i(t) t}^{4}+\beta_{12} \mathrm{X}^{10}+\beta_{13} \mathrm{X}^{6}{ }_{t(t) t}+u_{i(t) t} \\
& h_{t(t) t}=\left\{\begin{array}{l}
1 \text { se } h_{t(t) t}^{*}>0 \\
0 \text { se } h_{i(t) t}^{*} \leq 0
\end{array}, i(t)=1, \ldots, N, t=1, \ldots, T \text { e } u_{t(t) t} \sim N(0,1) .\right.
\end{aligned}
$$

sendo $\tau_{i(t) t}$ a variável endógena que identifica se o indivíduo trabalha no setor rural ou não, o vetor de variáveis independentes com índice sobrescrito 2 representa as característica maternas (status de saúde e educação); o vetor com índice 3 representa as características domiciliares (possui filtro e número de irmãos menores que 5 anos de idade e maiores de 16 anos de idade); com índice 4, a renda familiar (ln da renda per capita familiar exceto renda da criança); com índice 5, características individuais (sexo e raça); com índice 6, região e local de domicílio (residência urbana e regiões); com índice 7 , o ano e $\bar{\theta}_{\epsilon}^{*}$ são as binárias das coortes. $\mathrm{O}$ vetor que possui o índice 8 representa a educação da mãe, com índice 9 representa o número total de irmãos e, com índice 10, as características individuais (idade, sexo e raça).

Portanto, o procedimento adotado utiliza dados individuais e estima o primeiro estágio com base na eq. (8), sendo o trabalho infantil estimado em função das variáveis de controle tempo-invariantes e tempo-variantes e binárias de coortes. ${ }^{13}$ Em um segundo momento, se estabelece a regressão principal, no qual o status de saúde é estimado em função das binárias de coorte, a variável de trabalho e as variáveis de controle. Esse procedimento é elaborado no programa Stata 9.1 utilizando o comando ivprobit.

${ }_{13}$ As binárias de coorte são $\mathrm{D}_{510}$ igual a 1 para os indivíduos com 5 anos em 1998 e 10 anos em 2003, $\mathrm{D}_{611}$ igual a 1 para indivíduos com 6 anos em 1998 e 11 em 2003 e assim sucessivamente até $\mathrm{D}_{1520}$ igual a 1 para os indivíduos com 15 anos em 1998 e 20 anos em 2003. 
As outras equações propostas seguem a mesma lógica e variáveis acima expostas. Mais detalhes sobre as variáveis utilizadas estão descritos na sub-seção 2.4 dessa seção e na descrição dos resultados (ver Tabela 1).

\subsection{As variáveis selecionadas}

Nesta seção, serão descritas as variáveis das PNADs 1998 e 2003 que irão compor o modelo empírico. Iniciando pela variável dependente observa-se que, da mesma forma que a habilidade, o estoque de saúde é uma variável difícil de ser mensurada com acurácia. A PNAD fornece duas proxies, uma subjetiva e outra objetiva, que são os fluxos de saúde ${ }^{14}$. As medidas objetivas podem ser divididas em duas. A primeira medida objetiva é o critério médico, que questiona os indivíduos sobre a presença de doenças crônicas (12 tipos de doenças) e o número de dias que não pôde exercer atividades habituais. A segunda medida objetiva é funcional, questionando os indivíduos quanto a sua capacidade de realizar tarefas habituais (7 questões para a população de 14 anos ou mais).

Para a análise do impacto do trabalho infantil sobre a saúde, essas questões objetivas não são as mais adequadas. O critério funcional analisa somente os indivíduos acima de 14 anos, logo não pode ser utilizada neste trabalho. Julga-se que o critério médico não é um indicador adequado para o estudo de problemas de saúde de crianças e jovens, visto que é pouco provável - apesar de não ser impossível - que uma criança tenha adquirido problemas crônicos devido ao trabalho em um espaço temporal curto ${ }^{15}$.

O critério subjetivo é o da auto-avaliação do status de saúde individual. Nesse critério, o indivíduo respondente é questionado sobre seu status de saúde e dos outros membros do domicílio, classificando-os como: 1-muito bom, 2-bom, 3-regular, 4-ruim, 5-muito ruim. Essa é uma medida usual em pesquisas sobre saúde e consegue extrair o estado global não restrito a um período de referência (Andrade, 2002).

\footnotetext{
${ }^{14}$ Para análise detalhada das variáveis de saúde na PNAD 1998 ver Andrade (2002).

${ }^{15}$ Doenças crônicas referem-se às seguintes doenças: coluna, artrite, câncer, diabetes, bronquite, hipertensão, doença do coração, insuficiência renal crônica, depressão, hipertensão, tendinite e cirrose.
} 
O problema dessa variável, contudo, é que ela está condicionada a percepção individual e, portanto, indivíduos com estoques de saúde idênticos podem ter classificações distintas da auto-avaliação. Como exemplo, selecionando as pessoas que tinham câncer na PNAD 1998, aproximadamente $20 \%$ declararam ter status de saúde muito bom e bom.

Pelas vantagens apresentadas do critério subjetivo de auto-avaliação, mas ciente das restrições, esse será o critério empregado neste trabalho, criando a seguinte binária, status de saúde muito bom $=1$ (se status de saúde for "muito bom" =1) e status de saúde muito bom $=0$ (se status de saúde for "bom" $=2$ ou "regular" $=3$ ou "ruim" $=4$ ou “muito ruim” = 5). Considerou-se esse corte, já que mais de $90 \%$ dos indivíduos analisados possuem saúde muito boa e boa.

As crianças e jovens serão divididos entre aqueles que trabalham e que não trabalham. As crianças que trabalham são aquelas que possuíam trabalho na semana anterior à entrevista $(21$ a 27 de setembro de 2003), ou se produziam alimento para consumo próprio, ou trabalhavam na construção para o próprio uso, ou se trabalhavam, mas não estavam trabalhando naquela semana, por estarem de férias ou por terem problemas de saúde.

Além da variável que identifica se a criança trabalha ou não, foi criada uma segunda variável relativa ao trabalho infantil que identifica se o trabalho é de risco ou não. A classificação de trabalho de risco foi elaborada com base na ocupação dos indivíduos e ramo de atividade dos estabelecimentos, seguindo a classificação proposta por Kassouf et al. (2004), que utilizaram as informações do suplemento especial da PNAD 2001 sobre segurança no trabalho para indivíduos entre 5 e 17 anos de idade, medindo a proporção de machucados entre os trabalhadores dessa faixa etária. Em seguida, combinaram essas informações com a classificação de trabalho perigoso proibido para menores de 18 anos, elaborada pelo Ministério do Trabalho e Emprego. Dessa forma, os autores construíram uma relação de ocupações perigosas - e seus respectivos códigos de ocupação - que pode ser aplicada facilmente aos dados das PNADs ou Censos Demográficos. Para este estudo, foi utilizada essa relação para definir as ocupações perigosas das PNADs 1998 e 2001. 
O setor de atividade será dividido em agrícola e não-agrícola. Assim, surge a terceira variável relativa a trabalho que indica se o indivíduo trabalha no setor agrícola ou não, fornecendo o impacto das características do setor agrícola na saúde da criança. Como exemplo, de forma geral, acredita-se que as atividades agrícolas são mais fisicamente demandantes em relação às outras atividades e, portanto, podem ter maior impacto sobre a saúde da criança (Banco Mundial, 2001; Fassa et al., 2000). Essa é uma das hipóteses que serão testadas neste artigo.

As características maternas utilizadas foram os anos de estudo da mãe, que influenciam na eficiência da transformação de um bem comprado no mercado em saúde e o status de saúde materna (status de saú$\underline{\text { de bom }=1}$ se status for "muito bom" e "bom" e 0 se "regular", "ruim" e "muito ruim"), podendo controlar características genéticas.

As características domiciliares utilizadas foram: existência de filtro de água e o número de irmãos menores de 5 anos e maiores que 16. Foi escolhido esse corte etário, pois pessoas com mais de 16 anos podem contribuir trabalhando ou auxiliando nos serviços domésticos (limpando, cuidando dos irmãos menores etc). Já as crianças com cinco anos ou menos demandam a presença e atenção maior e sempre estão dentro de casa sendo mais vulneráveis a doenças e consecutiva disseminação.

Para controle do investimento em saúde serão utilizados: o logaritmo neperiano da renda total per capita familiar exceto a renda da criança, que representa a capacidade de investimento ${ }^{16}$.

As variáveis de controle individual utilizadas e que podem influenciar no estoque e na depreciação do estoque de saúde são sexo e raça (branco ou não branco). Não foi considerada a idade, visto que essa teria alta correlação com as binárias das coortes de nascimento.

A região e local do domicílio, também podem afetar o status de saú-

\footnotetext{
${ }^{16}$ Os valores monetários foram deflacionados pelo INPC (Índice Nacional de Preços ao Consumidor) para setembro de 2003. Optou-se pelo INPC, já que esse é um índice de custo de vida e pode representar de forma mais adequada a capacidade total de investimento da família, ou seja, contempla todos os grupos de bens e serviços consumidos pelas famílias. Caso fosse utilizado gasto em saúde, seria mais adequado utilizar outro índice para deflacionar.
} 
de do indivíduo, pelo acesso aos serviços de saúde. ${ }^{17}$ Como variáveis de controle demográfico, serão utilizadas o local de residência - urbano ou rural - e a região que se localiza o domicílio (Norte, Nordeste, Sul, Sudeste, Centro-Oeste).

\section{Resultados e discussão}

\subsection{Estatísticas descritivas}

A amostra da PNAD de 1998 contém um total de 7.240 crianças entre 5 e 15 anos de idade trabalhando. No ano de 2003 tem-se um total de 16.960 trabalhadores de 10 a 20 anos na amostra.

A amostra foi elaborada levando em conta a existência de uma família nuclear ${ }^{18}$. Esse critério não prejudicou o grupo dos indivíduos de 1998, no entanto, observa-se um forte decréscimo de pessoas no total de 2003. Esse fato pode gerar viés nas estimativas, já que estão sendo selecionados indivíduos com características bem específicas, ou seja, indivíduos com 20 anos que moram com os pais ou dependam deles, por exemplo. Acredita-se que esse problema é menor em relação ao problema de selecionar indivíduos que moravam com os pais em 1998 e são chefes de família em 2003, principalmente no que se refere à decisão de alocação dos recursos familiares.

Ao analisar o status de saúde, observa-se que pouquíssimas crianças e jovens consideram sua saúde muito ruim ou ruim - menos de $1 \%$ - e aproximadamente $7 \%$ consideram regular (Figura 1) ${ }^{19}$. Esse padrão se mantém no grupo analisado no tempo, sendo a principal mudança a

\footnotetext{
${ }^{17}$ O governo possui dispêndios e diversos programas de saúde que contribuem substancialmente para a saúde das pessoas carentes e que são distintos entre as regiões. Dentre eles estão o Piso de Atenção Básica (PAB) e o Programa de Saúde da Família (PSF), respectivamente. Incorporar esses valores na regressão fica estatisticamente complicado devido ao peso fornecido pela PNAD e utilizado na regressão. Além disso, tem-se uma média no estado o que não necessariamente reflete o município onde está o indivíduo, assim optou-se pela utilização de binárias regionais.

${ }^{18}$ Família nuclear é aquela que possui pai, mãe e filhos.

${ }^{19}$ De maneira geral, o respondente é uma pessoa do domicílio, em geral, o chefe, que responde as questões para todos os membros da família.
} 
diminuição daqueles que consideram sua saúde muito boa e aumento dos que consideram sua saúde boa.

A Figura 1 mostra ainda que há um maior número de indivíduos que classificam seu status de saúde como regular e bom no grupo dos que trabalham em relação aos que não trabalham. Ou seja, os indivíduos que não trabalham possuem maior probabilidade de classificar seu status de saúde como muito bom em relação àqueles que trabalham. Essa probabilidade diminui de 1998 para 2003, tanto para aqueles que trabalham como para os que não trabalham. Essa diminuição pode indicar a depreciação natural do estoque de saúde dos indivíduos.

Se analisados somente os indivíduos que trabalham, os setores de ocupação que apresentam a maior quantidade de indivíduos em 1998 com saúde regular são o de serviços e de construção (ver Figura 2). No entanto, em 2003 esse diferencial se altera, sendo os setores agrícola e comércio os que possuem o maior número de indivíduos com saúde regular. Os setores serviço e construção são aqueles que possuem o menor percentual de indivíduos com classificação muito boa do estado de saúde em 1998 e 2003.

Observa-se que ocupações distintas impõem diferente depreciação ao estoque de saúde. Assim, torna-se necessário não somente analisar o trabalho, mas se esse é de risco ou não. Focando no trabalho de risco, em 1998, o setor de serviços possuía o maior percentual de indivíduos, seguido pelo agrícola e industrial. No período seguinte, observa-se que indústria e comércio ganham espaço do setor de serviços, de forma que agropecuária e indústria são as áreas que mais empregam indivíduos em atividade perigosa em 2003 (Figura 3). Nota-se também que a agropecuária é o setor que mais emprega crianças e jovens em atividades não-perigosas, tanto em 1998 como em 2003. Observa-se que, em 2003, há indivíduos com mais de 18 anos que, por lei, podem ser contratados para alguns tipos de trabalhos de risco. 

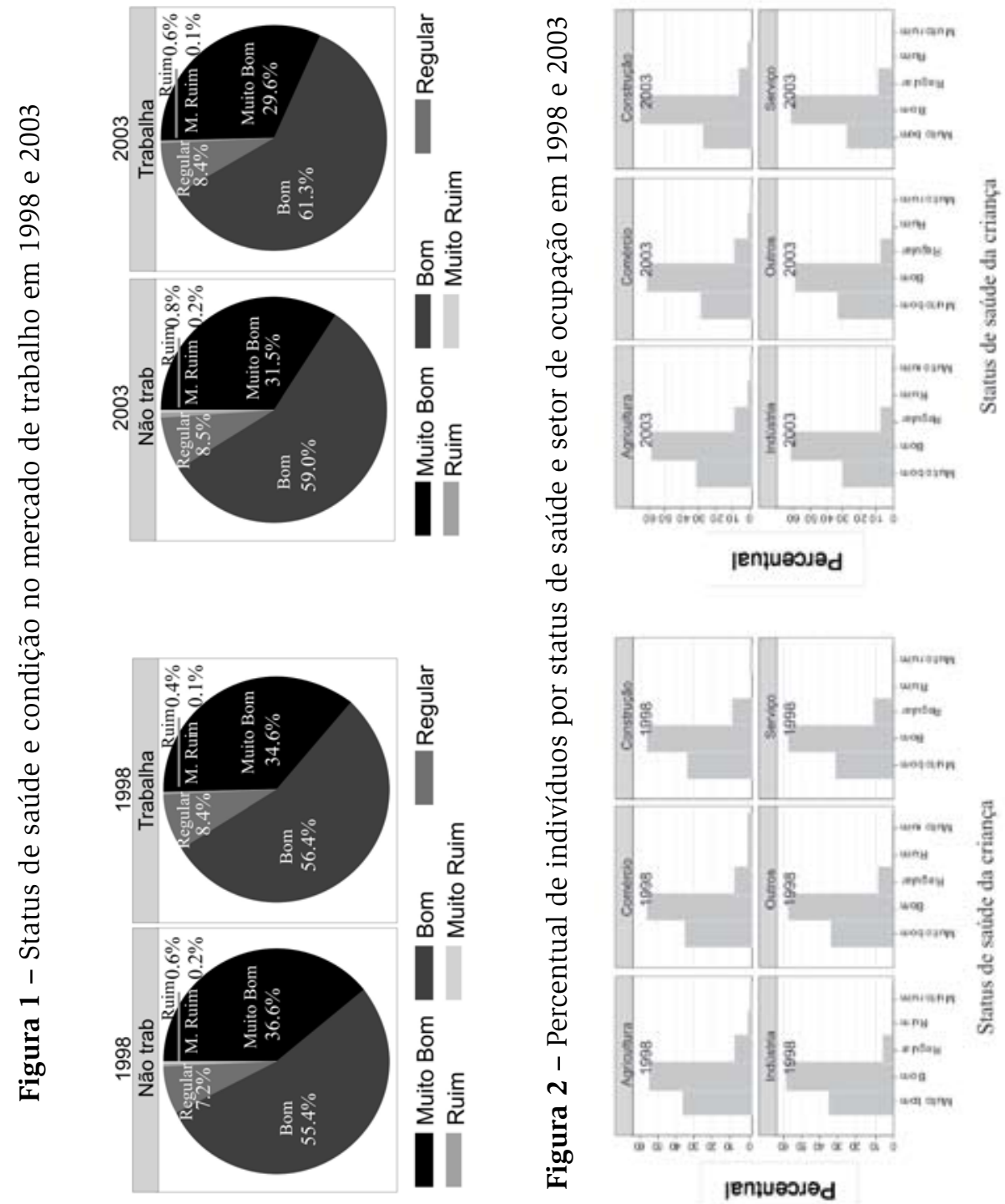

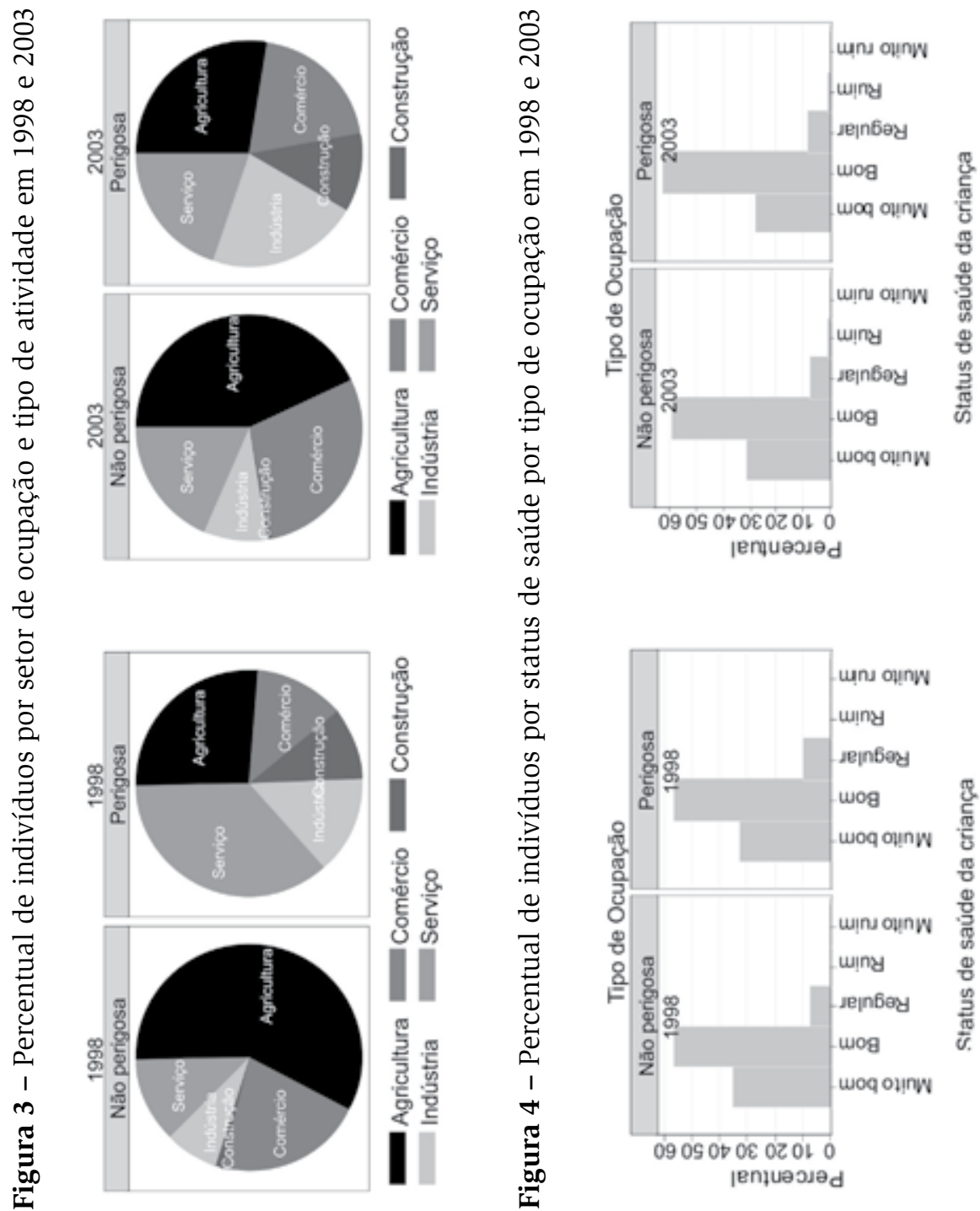
Se analisado a relação saúde e educação, percebe-se a presença de uma correlação negativa entre o status de saúde e o atraso escolar para os jovens entre 10 e 15 anos em 1998 e entre 15 e 20 em 2003 (utilizouse essa faixa etária pelo fato de a idade obrigatória de ingresso na escola ser de sete anos e por conta do tamanho da amostra). Entretanto, essa correlação é mais forte entre os jovens que não estão trabalhando, principalmente em 2003 (Figura 5).

Figura 5 - Média do atraso escolar e status de saúde por status de ocupação em 1998 e 2003
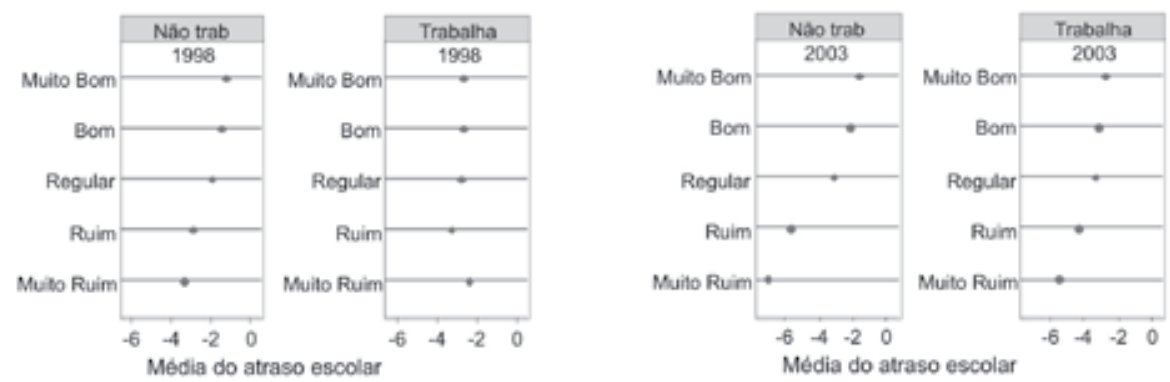

As médias e variâncias das variáveis utilizadas nas equações estão na Tabela A1 do anexo.

\subsection{Resultados econométricos}

Os resultados econométricos da modelagem de pseudo-painel, utilizando o modelo probit com variável independente endógena é apresentado na Tabela 1, que contém os efeitos marginais dos coeficientes estimados. A segunda coluna representa a $1^{\text {a }}$ equação, que contém somente os indivíduos que trabalham. A terceira coluna contém a $2^{\mathrm{a}}$ equação com aqueles indivíduos que exercem atividades perigosas. A quarta e a quinta colunas representam a $3^{\mathrm{a}}$ e $4^{\mathrm{a}}$ equações com os indivíduos que moram no meio rural e no meio urbano, respectivamente.

Foram utilizadas duas variáveis para analisar o impacto do trabalho infantil na saúde. A primeira é se o indivíduo exerce atividade no setor agrícola ou não. Essa é utilizada na $1^{\mathrm{a}}$ e $2^{\mathrm{a}}$ equações. A segunda 
variável para essa análise é se o indivíduo trabalha ou não, utilizada na $3^{\mathrm{a}}$ e $4^{\mathrm{a}}$ equações.

Ao separar somente aqueles indivíduos que trabalham, observa-se que o setor no qual o indivíduo exerce sua atividade não tem influência sobre a saúde. Assim, trabalhar no meio rural possui impacto positivo, mas não significativo na saúde da criança. Pelo senso comum, as condições de trabalho no campo são inferiores às da cidade, levando a um pior status de saúde dos indivíduos. Imagina-se sempre crianças trabalhando em campos de sisal, cana, carvão etc. No entanto, o resultado obtido não confirma essa hipótese. De forma análoga, exercer trabalho de risco no meio rural ( $2^{\mathrm{a}}$ equação), apesar de possuir um impacto negativo sobre a saúde, não é significativo, ou seja, é indiferente em termos do status de saúde o indivíduo exercer atividades perigosas no campo ou na cidade.

Ao selecionar somente aqueles indivíduos que moram no meio rural ( $3^{\mathrm{a}}$ equação), o fato de o indivíduo trabalhar tem impacto positivo, mas não significativo. Assim, para aqueles que moram no meio rural não há evidências de que trabalhar ou não trabalhar afeta o status de saúde individual. No entanto, se o indivíduo residir no meio urbano ( $4^{\mathrm{a}}$ equação) o fato de trabalhar diminui a probabilidade de o indivíduo ter status de saúde muito bom em 0,128 ponto percentual, sendo esse resultado significativo a 5,7\%. Portanto, o trabalho exercido por aqueles indivíduos que moram no meio urbano penaliza mais o status de saúde das crianças do que daqueles indivíduos que moram no meio rural. Apesar de um resultado surpreendente, pode não ser equivocado. A primeira hipótese para esse resultado é que as crianças no meio rural, na média, podem exercer suas atividades mais próximas de seus pais, obtendo dos mesmos um grau de atenção maior e possivelmente são menos expostas a situações de risco.

Por outro lado, é possível que parte dos resultados das duas equações seja devida à capacidade das pessoas que trabalham no meio rural de suportar mais ou de achar normal determinados problemas de saúde. Essa hipótese parece razoável, uma vez que neste trabalho considerase o status de saúde auto-avaliado como variável dependente. Observe que ambas são hipóteses não testadas neste estudo, sendo temas para futuro desenvolvimento. 
As características da mãe utilizadas nas quatro equações foram o status de saúde materno e a educação materna. Essas duas variáveis apresentaram o sinal positivo, conforme esperado. A variável de status de saúde da mãe mostrou-se importante em todas as equações para a determinação da saúde do filho. Observa-se que o fato de a mãe possuir status de saúde muito bom e bom aumenta em aproximadamente 0,3 ponto percentual a probabilidade de o filho possuir status de saúde muito bom nas quatro equações.

A educação da mãe, por sua vez, não se mostrou tão importante quanto a sua saúde. Essa variável foi significativa somente para a equação 4, que representa somente os indivíduos que moram na cidade e para a equação 1, que reúne somente aqueles que trabalham. Provavelmente, essa não significância do meio rural pode ser devido ao enorme contingente de mães com baixa educação formal. Aproximadamente $49 \%$ das mães possuem até dois anos de estudo no meio rural contra $20 \%$ no meio urbano.

As variáveis de características domiciliares analisadas foram a presença de filtro e número de irmãos menores de 5 e maiores de 16 anos. A presença do filtro no domicílio tem impacto positivo, como esperado, e significativo para a maior parte das equações (exceto para a $3^{\mathrm{a}}$ ). Observa-see que o fato de possuir filtro em casa aumenta de 0,067 ( $4^{\mathrm{a}}$ eq.) para 0,135 ( $2^{\mathrm{a}}$ eq.) ponto percentual a probabilidade de a criança possuir status de saúde muito bom.

As variáveis de número de irmãos mostraram o sinal conforme esperado na maior parte das equações, ou seja, esperava-se que irmãos pequenos tivessem impactos negativos, pois a presença de irmãos pequenos intensifica a convivência e a disseminação de doenças, além de demandar grande atenção por parte da mãe. Já irmãos maiores teriam impactos positivos, já que ficariam mais ausentes da casa, podendo colaborar para o rendimento familiar ou nos cuidados despendidos aos outros irmãos. No entanto, essas variáveis só foram significativas para a $4^{\mathrm{a}}$ equação. Assim, a presença de um irmão com menos de 5 anos de idade na família daqueles indivíduos que residem no meio urbano, diminui em 0,04 ponto percentual a probabilidade de a criança possuir status de saúde muito bom.

A renda familiar per capita, exceto a renda da criança (em logaritmo), teve sinal esperado e mostrou-se significativa em todas 
as equações. Essa é uma variável que mede a capacidade de investimento da família. Observa-se que aumentos de $1 \%$ nessa renda aumenta em 0,127 ponto percentual a probabilidade de a criança ter saúde muito boa. Esse impacto não é tão expressivo para aqueles que residem no campo, sendo o aumento na probabilidade de 0,048 ponto percentual. Para os indivíduos que trabalham e para aqueles que trabalham em atividades perigosas, o impacto está ao redor de 0,1 ponto percentual.

As características das crianças utilizadas nas equações foram raça e sexo. A variável sexo foi ambígua em relação ao sinal nas regressões, sendo significativa somente para aqueles indivíduos que trabalham, no qual o fato de ser homem impacta positivamente na saúde. No entanto, a variável raça mostrou-se quase sempre positiva e foi significativa para a $3^{\mathrm{a}}$ e $4^{\mathrm{a}}$ equação. $\mathrm{O}$ fato de ser branco aumenta em 0,053 e 0,068 ponto percentual a probabilidade de ter boa saúde. Como educação e renda estão controladas, o fato de ser branco pode estar indicando uma facilidade de acesso ao sistema de saúde por esses indivíduos, por residirem em bairros mais ricos e próximos aos centros de atendimento. Assim, o tempo de acesso aos não-brancos torna-se elevado, dificultando ou encarecendo o atendimento. Além disso, uma parte desses resultados pode ser devido às características genéticas e culturais relativas às diferentes raças.

As variáveis de localização de domicílio utilizadas foram: se esse está localizado no meio urbano ou não $\left(1^{\mathrm{a}}\right.$ e $2^{\mathrm{a}}$ equações) e qual a região do domicílio (Norte, Nordeste, Sul e Centro-Oeste). O impacto de morar no meio urbano foi ambíguo em relação ao sinal e não significativo para as equações dos indivíduos que trabalham e que trabalham em atividades perigosas.

Entretanto, as pessoas que moram na região Sudeste do Brasil têm chances maiores de status de saúde muito bom em relação às que moram em outras regiões. Observa-se que a região Norte é que mais tem impacto na saúde - dessa forma, caso a residência seja nessa região, há a diminuição da probabilidade de a criança apresentar status de saúde muito bom de 0,459 a 0,693 ponto percentual. 
Tabela 1 - Resultados do modelo probit com variável endógena para as quatro equações.

\begin{tabular}{|c|c|c|c|c|}
\hline Saúde muito boa & $\begin{array}{l}\text { Indivíduos } \\
\text { que trabalham }\end{array}$ & $\begin{array}{l}\text { Trabalho em } \\
\text { atividades } \\
\text { perigosas }\end{array}$ & $\begin{array}{l}\text { Indivíduos que } \\
\text { vivem no meio } \\
\text { rural }\end{array}$ & $\begin{array}{l}\text { Indivíduos que } \\
\text { vivem no meio } \\
\text { urbano }\end{array}$ \\
\hline \multirow{2}{*}{$\begin{array}{l}\text { Trabalha setor } \\
\text { agrícola }\end{array}$} & 0,393 & $-0,050$ & - & - \\
\hline & $(1,42)$ & $(-0,11)$ & - & - \\
\hline \multirow{2}{*}{ Trabalha } & - & - & 0,228 & $-0,128$ \\
\hline & - & - & $(1,12)$ & $(-1,9) * * *$ \\
\hline \multirow{2}{*}{ Saúde da mãe } & 0,326 & 0,315 & 0,343 & 0,350 \\
\hline & $(12,29)^{*}$ & $(7,78)^{*}$ & $(11,8)^{*}$ & $(28,98)^{*}$ \\
\hline \multirow{2}{*}{ Educação da mãe } & 0,010 & 0,010 & 0,003 & 0,014 \\
\hline & $(2,53)^{* *}$ & $(1,6)$ & $(0,57)$ & $(8,84)^{*}$ \\
\hline \multirow{2}{*}{ Possui filtro } & 0,109 & 0,135 & 0,011 & 0,067 \\
\hline & $(3,94)^{*}$ & $(3,16)^{*}$ & $(0,36)$ & $(5,94)^{*}$ \\
\hline \multirow{2}{*}{$\begin{array}{l}\text { Número de irmãos } \\
\text { menores de } 5 \text { anos }\end{array}$} & $-0,026$ & $-0,012$ & $-0,029$ & $-0,042$ \\
\hline & $(-0,98)$ & $(-0,31)$ & $(-1.3)$ & $(-4,41)^{*}$ \\
\hline \multirow{2}{*}{$\begin{array}{l}\text { Número de irmãos } \\
\text { maiores de } 16 \text { anos }\end{array}$} & 0,008 & 0,001 & $-0,006$ & 0,010 \\
\hline & $(0,61)$ & $(0,07)$ & $(-0,39)$ & $(1,66) * * *$ \\
\hline \multirow{2}{*}{$\begin{array}{l}\text { Renda per capita } \\
\text { (exceto criança - log) }\end{array}$} & 0,094 & 0,108 & 0,048 & 0,127 \\
\hline & $(4,57)^{*}$ & $(3,49)^{*}$ & $(2,42)^{* *}$ & $(18,98)^{*}$ \\
\hline \multirow{2}{*}{ Homem } & 0,071 & 0,069 & $-0,016$ & $-0,006$ \\
\hline & $(1,96)^{* * *}$ & $(1,1)$ & $(-0,36)$ & $(-0,56)$ \\
\hline \multirow{2}{*}{ Branco } & 0,026 & $-0,013$ & 0,053 & 0,068 \\
\hline & $(0,93)$ & $(-0,31)$ & $(1,73) * * *$ & $(5,97)^{*}$ \\
\hline \multirow{2}{*}{ Urbano } & 0,227 & $-0,071$ & - & \\
\hline & $(1,35)$ & $(-0,27)$ & - & \\
\hline \multirow{2}{*}{ Norte } & $-0,617$ & $-0,693$ & $-0,502$ & $-0,459$ \\
\hline & $(-10,58)^{*}$ & $(-7,18)^{*}$ & $(-4,29)^{*}$ & $(-23,21)^{*}$ \\
\hline \multirow{2}{*}{ Nordeste } & $-0,093$ & $-0,144$ & 0,033 & $-0,093$ \\
\hline & $(-2,81)^{*}$ & $(-2,16)^{* *}$ & $(0,89)$ & $(-7)^{*}$ \\
\hline \multirow{2}{*}{ Sul } & $-0,144$ & $-0,052$ & $-0,160$ & $-0,120$ \\
\hline & $(-3,58)^{*}$ & $(-0,83)$ & $(-2,59)^{*}$ & $(-7,7)^{*}$ \\
\hline \multirow{2}{*}{ Centro- Oeste } & $-0,274$ & $-0,279$ & $-0,048$ & $-0,231$ \\
\hline & $(-6,77)^{*}$ & $(-4,6)^{*}$ & $(-1,08)$ & $(-14,46)^{*}$ \\
\hline \multirow{2}{*}{ D611* } & $-0,074$ & 0,051 & $-0,043$ & $-0,020$ \\
\hline & $(-0,55)$ & $(0,26)$ & $(-0,71)$ & $(-0,85)$ \\
\hline \multirow{2}{*}{ D712* } & $-0,057$ & $-0,150$ & $-0,095$ & $0,004 w$ \\
\hline & $(-0,46)$ & $(-0,8)$ & $(-1,54)$ & $(0,18)$ \\
\hline
\end{tabular}


Tabela 1 - (continuação)

\begin{tabular}{|c|c|c|c|c|}
\hline Saúde muito boa & $\begin{array}{l}\text { Indivíduos } \\
\text { que trabalham }\end{array}$ & $\begin{array}{c}\text { Trabalho em } \\
\text { atividades } \\
\text { perigosas }\end{array}$ & $\begin{array}{l}\text { Indivíduos que } \\
\text { vivem no meio } \\
\text { rural }\end{array}$ & $\begin{array}{c}\text { Indivíduos que } \\
\text { vivem no meio } \\
\text { urbano }\end{array}$ \\
\hline \multirow{2}{*}{ D813* } & $-0,169$ & $-0,121$ & $-0,139$ & 0,010 \\
\hline & $(-1,4)$ & $(-0,72)$ & $(-2,22)^{* *}$ & $(0,41)$ \\
\hline \multirow{2}{*}{ D914* } & $-0,061$ & $-0,108$ & $-0,096$ & 0,007 \\
\hline & $(-0,51)$ & $(-0,66)$ & $(-1,47)$ & $(0,3)$ \\
\hline \multirow{2}{*}{ D1015* } & $-0,036$ & $-0,009$ & $-0,139$ & $-0,012$ \\
\hline & $(-0,32)$ & $(-0,06)$ & $(-1,95)^{* * *}$ & $(-0,51)$ \\
\hline \multirow{2}{*}{ D1116* } & $-0,041$ & $-0,138$ & $-0,081$ & $-0,039$ \\
\hline & $(-0,37)$ & $(-0,89)$ & $(-1,04)$ & $(-1,54)$ \\
\hline \multirow{2}{*}{ D1217* } & $-0,039$ & $-0,069$ & $-0,148$ & 0,003 \\
\hline & $(-0,34)$ & $(-0,44)$ & $(-1,66) * * *$ & $(0,12)$ \\
\hline \multirow{2}{*}{ D1318* } & $-0,054$ & $-0,027$ & $-0,196$ & $-0,012$ \\
\hline & $(-0,46)$ & $(-0,17)$ & $(-2,02) * *$ & $(-0,43)$ \\
\hline \multirow{2}{*}{ D1419* } & $-0,079$ & 0,017 & $-0,291$ & $-0,024$ \\
\hline & $(-0,65)$ & $(0,11)$ & $(-2,73)^{*}$ & $(-0,75)$ \\
\hline \multirow{2}{*}{ D1520* } & $-0,057$ & $-0,025$ & $-0,238$ & $-0,023$ \\
\hline & $(-0,47)$ & $(-0,15)$ & $(-2)^{* *}$ & $(-0,67)$ \\
\hline \multirow{2}{*}{ Ano } & $-0,135$ & $-0,256$ & $-0,233$ & $-0,120$ \\
\hline & $(-3,56)^{*}$ & $(-5,08)^{*}$ & $(-4,23)^{*}$ & $(-7,51)^{*}$ \\
\hline \multirow{2}{*}{ Constante } & $-1,346$ & $-0,996$ & $-0,696$ & $-1,261$ \\
\hline & $(-3,99) *$ & $(-2,17) * *$ & $(-7,14)^{*}$ & $(-32,64) *$ \\
\hline $\begin{array}{l}\text { Número de } \\
\text { observações }\end{array}$ & 13777 & 5971 & 10887 & 83342 \\
\hline Wald $\chi^{2}$ & 495,85 & 247,95 & 262,80 & 4125,60 \\
\hline $\operatorname{Pr}>\chi^{2}$ & 0,000 & 0,000 & 0,000 & 0,000 \\
\hline
\end{tabular}

Nota: Entre parênteses estão os valores da estatística t. * significativo a $1 \%$; ${ }^{* *}$ significativo a $5 \%$ $\mathrm{e}^{* * *}$ significativa a $10 \%$.

\section{Conclusão}

O objetivo deste artigo foi verificar se o trabalho infantil exercido no setor agrícola afeta mais a saúde da criança do que o trabalho exercido em outros setores. O entendimento dessa relação é importante, visto que pode fornecer subsídios para o combate ao trabalho infantil e permitir o direcionamento de políticas para restabelecer a saúde da criança em casos no qual a erradicação não foi efetivada. 
Para a análise, foram utilizados dois anos da Pesquisa Nacional por Amostra de Domicílios (PNAD), 1998 e 2003, que trazem suplemento especial sobre saúde. Como essas duas pesquisas não caracterizam um painel, a técnica econométrica empregada foi a de pseudo-painel, sendo possível controlar os efeitos individuais. As estimativas foram elaboradas utilizando o modelo probit com variável independente endógena. Embora a literatura nacional e internacional apresente poucas pesquisas nessa área, os resultados obtidos foram consistentes com aqueles alcançados anteriormente.

Para a análise do trabalho infantil sobre a saúde foram utilizadas quatro equações. A primeira verifica se o fato de trabalhar no setor agrícola possui impactos distintos dos outros indivíduos que trabalham em outros setores. A segunda é similar a primeira, mas considera somente aqueles indivíduos que trabalham em atividades de risco. A terceira analisa se trabalhar afeta a saúde das crianças que moram no campo e a quarta equação analisa essa relação para o meio urbano.

Os resultados mostram que o trabalho no campo não causa impactos distintos na saúde da criança em relação a outros setores de atividades. Esse resultado não corrobora o senso comum de que as condições de trabalho no campo são piores que na cidade. Da mesma forma, o impacto do trabalho infantil na saúde da criança é independente do setor no qual exerce uma atividade, sendo a atividade de risco ou não.

Para aqueles indivíduos que moram no meio rural, trabalhar ou não trabalhar não afeta o status de saúde individual. No entanto, se o indivíduo residir no meio urbano, trabalhar impacta negativamente na saúde da criança.

Esses resultados indicam que as políticas públicas de erradicação e de saúde ligadas à criança devem ser distintas entre os meios urbanos e rurais. Além disso, essas políticas devem ser regionalizadas. Em paralelo e setorialmente, melhoria no acesso ao sistema de saúde, garantia de acesso a medicamentos e promoção da educação materna em saúde devem ser incentivadas, visto que são aparentemente eficazes para aumentar o estoque de saúde da criança.

Neste artigo, a variável dependente utilizada foi status de saúde autoavaliado, sendo essa condicionada à percepção individual, de modo que indivíduos com mesmo estoque de saúde podem ter classificações distintas da sua saúde. Assim, a busca por uma variável menos dependente da 
percepção pode trazer benefícios para a análise dessa relação. Outro ponto importante é que uma análise mais detalhada das diferentes ocupações rurais pode trazer contribuições significativas para essa discussão, pois os resultados aqui apresentados são gerais nesse aspecto.

\section{Referências bibliográficas}

ALSAN, M.; BLOOM, D.E.;CANNING, D. The effect of population health on foreign direct investment. Cambridge: NBER, 2004. 25p. (Working Paper, 10596)

ANDRADE, M.V. A Saúde na PNAD. Belo Horizonte: UFMG/Cedeplar, 2002. 31p. (Texto para Discussão, 170).

BALAND, J.M.; ROBINSON, J.A. Is child labor inefficient? Journal of Political Economy, Chicago, v.108, n.4, p.663-679, Aug 2000.

BANCO MUNDIAL. Brazil: eradicating child labor in Brazil. Human Development Department, 2001. (Report, 21858-BR). Disponível em: $<$ http//www-wds.wolrdbank.org/external/lac > .

BASU, K. Child labor: cause, consequence, and cure, with remarks on international labor standards. Journal of Economic Literature, Nasheville, v.37, n.3, p.1083-1119, Sep. 1999.

BECKER, G.S.; MULLIGAM, C.B. The endogenous determination of time preference. The Quarterly Journal of Economics, Cambridge, v.112, n.3, p.729-758, Aug. 1997.

BROWNING, M; DEATON, A.; IRISH, M. A profitable approach to labor supply and commodity demands over the life-cycle. Econometrica, Evanston, v.53, n.3, p.503-544, May 1985.

CROPPER, M.L. Health, investment in health, and occupational choice. Journal of Political Economy, Chicago, v.85, n.6, p.1273-1294, Dec 1977.

DEATON, A. Panel data from time series of cross-sections. Journal of Econometrics, Amsterdan, v.30, n.1, p.109-126, Oct. 1985.

DEATON, A. The analysis of household surveys. 1.ed. Baltimore: The Johns Hopkins University Press, 1997. 488p. 
EMERSON, P.M.; PORTELA, A. Bargaining over sons and daughters: child labor, school attendance and intra-household gender bias in Brazil. Nashville: Vanderbilt University, 2001. 44p. (Working Paper , 0213).

FASSA, A.G.; FACCHINI, L.A.; DALL'AGNOL, M.M.; CHRISTIANI, D. Child labor and health: problems and perspective. Boston: Harvard School of Public Health, 1999. 17 p. (Takemi Program in International Health Research Paper, 160).

FOGEL, R.W. Health, nutrition and economic growth. Economic Development and Cultural Change, Chicago, v.52, n.3, p.643-658, Apr. 2004.

FRANCAVILLA, F.; LYON, S. Household chores and child health: preliminary evidence from six countries. Florence: Innocenti Research Centre, 2003. 18p. (Understanding Children's Work Discussion Paper, draft).

GROSSMAN, M. On the concept of health capital and demand for health. Journal of Political Economy, Chicago, v.80, n.2, p.235-255, Jul. 1972 .

GROSSMAN, M. The human capital model. In: A.J.Culyer; J.P.Newhouse, ed., Handbook of health economics. v.1. Elsevier Science: Amsterdam, 2000. 936p.

GUIFFRIDA, A.; IUNES, R.F.; SAVEDOFF, W.D. Health and poverty in Brazil: Estimation by structural equation model with latent variables. Washington: Inter-American Development Bank. Oct. 2001. 30p. (mimeo).

INSTITUTO BRASILEIRO DE GEOGRAFIA E ESTATÍSTICA. Pesquisa nacional de amostra por domicílios: 2003. Rio de Janeiro: IBGE, 2005.

INSTITUTO BRASILEIRO DE GEOGRAFIA E ESTATÍSTICA. Pesquisa nacional de amostra por domicílios: 1998. Rio de Janeiro: IBGE, 2000.

KASSOUF, A.L. Aspectos sócio-econômicos do trabalho infantil no Brasil. Brasília: Ministério da Justiça, 2002. 124p.

KASSOUF, A.L.; MCKEE, M.; MOSSIALOS, E. Early entrance to the job market and its effects on adult health: Evidence from Brazil. Health Policy and Planning, Oxford, v.16, n.1, p.21-28, Mar 2001. 
LEVISON, D. Children as economic agents. Feminist Economics, Richmond, v.6, n.1, p.125-134, Mar 2000.

MENEZES-FILHO, N.A.Microeconometria.In:M.B.Lisboa; N.A.MenezesFilho, ed., Microeconomia e sociedade no Brasil. Contra Capa: Rio de Janeiro, 2001. 470p.

MOFFITT, R. Identification and estimation of dynamic models with a time series of repeated cross-sections. Journal of Econometrics, Amsterdan, v.59, n.1, p.99-123, Sep. 1993.

MUURINEN, J. Demand for health: a generalized Grossman model. Journal of Health Economics, Amsterdam, v.1, n.1, p.5-28, May 1982.

O'DONNELL, O.; ROSATI, F.C.; VAN DOORSLAER, E. Child labour and health: Evidence and research issues. Florence: Innocenti Research Centre, 2002. 31p. (Understanding Children's Work Discussion Paper, draft)

PERES, A.; BENEDICTO, N. A caminho da escola: 10 anos de luta pela erradicação do trabalho infantil no Brasil. Rio de Janeiro: Instituto Souza Cruz, 2003. 132p.

RAY, R. How child labour and child schooling interact with adult labour. Washington D.C.: The World Bank, 1999. 39p. (Working Paper Series, 0968).

ROSATI, F.; STRAUB, R. Does work during childhood affect adult's health? An analysis for Guatemala. Florence: Innocenti Research Centre, 2004. 24p. (Understanding Children's Work Discussion Paper, draft).

SOHN, B. How much has health improvement contributed to the Korean economic growth. 2000. 115 p. Tese (Doutorado) - Brown University, Providence, 2000.

STRAUSS, J.; THOMAS, D. Health, nutrition, and economic development. Journal of Economic Literature, Nasheville, v.36, n.2, p.766-817, Jun. 1998.

WOOLDRIDGE, J.M. Econometric analysis of cross section and panel data. 1.ed. Cambridge: MIT Press, 2002. 750p. 


\section{Anexo}

Tabela A1 - Médias e desvios padrões para as variáveis utilizadas nas quatro equações.

\begin{tabular}{|c|c|c|c|c|c|c|c|c|c|}
\hline \multirow[t]{2}{*}{ Variáveis } & & \multicolumn{2}{|c|}{$\begin{array}{l}\text { Somente } \\
\text { quem } \\
\text { trabalha }\end{array}$} & \multicolumn{2}{|c|}{$\begin{array}{l}\text { Somente } \\
\text { trabalho de } \\
\text { risco }\end{array}$} & \multicolumn{2}{|c|}{$\begin{array}{l}\text { Reside no } \\
\text { meio rural }\end{array}$} & \multicolumn{2}{|c|}{$\begin{array}{l}\text { Reside no } \\
\text { meio urbano }\end{array}$} \\
\hline & & 1998 & 2003 & 1998 & 2003 & 1998 & 2003 & 1998 & 2003 \\
\hline \multirow{2}{*}{ Trabalha } & Média & 1,000 & 1,000 & 1,000 & 1,000 & 0,244 & 0,445 & 0,061 & 0,211 \\
\hline & D.P. & 0,000 & 0,000 & 0,000 & 0,000 & 0,429 & 0,497 & 0,239 & 0,408 \\
\hline \multirow{2}{*}{$\begin{array}{l}\text { Trabalha setor } \\
\text { agrícola }\end{array}$} & Média & 0,548 & 0,313 & 0,373 & 0,251 & 0,215 & 0,367 & 0,011 & 0,022 \\
\hline & D.P. & 0,498 & 0,464 & 0,484 & 0,434 & 0,411 & 0,482 & 0,102 & 0,147 \\
\hline \multirow{2}{*}{ Saúde da mãe } & Média & 0,579 & 0,602 & 0,569 & 0,575 & 0,652 & 0,587 & 0,713 & 0,672 \\
\hline & D.P. & 0,494 & 0,490 & 0,495 & 0,494 & 0,476 & 0,492 & 0,452 & 0,470 \\
\hline \multirow{2}{*}{ Possui filtro } & Média & 0,442 & 0,485 & 0,465 & 0,461 & 0,444 & 0,434 & 0,570 & 0,520 \\
\hline & D.P. & 0,497 & 0,500 & 0,499 & 0,498 & 0,497 & 0,496 & 0,495 & 0,500 \\
\hline \multirow{2}{*}{ Educação da mãe } & Média & 3,190 & 4,671 & 3,381 & 4,100 & 2,884 & 2,912 & 6,128 & 6,420 \\
\hline & D.P. & 3,180 & 4,019 & 3,098 & 3,540 & 2,955 & 2,991 & 4,195 & 4,354 \\
\hline \multirow{2}{*}{$\begin{array}{l}\text { Número de irmãos } \\
\text { menores de } 5 \text { anos }\end{array}$} & Média & 0,472 & 0,179 & 0,396 & 0,185 & 0,647 & 0,301 & 0,409 & 0,197 \\
\hline & D.P. & 0,802 & 0,501 & 0,720 & 0,503 & 0,886 & 0,652 & 0,693 & 0,505 \\
\hline \multirow{2}{*}{$\begin{array}{l}\text { Número de irmãos } \\
\text { maiores de } 16 \text { anos }\end{array}$} & Média & 0,836 & 0,992 & 0,848 & 0,996 & 0,644 & 0,977 & 0,485 & 0,743 \\
\hline & D.P. & 1,085 & 1,089 & 1,088 & 1,121 & 1,064 & 1,192 & 0,873 & 0,966 \\
\hline \multirow{2}{*}{$\begin{array}{l}\text { Renda per capita } \\
\text { (exceto criança - log) }\end{array}$} & Média & 4,468 & 4,808 & 4,617 & 4,741 & 4,236 & 4,249 & 5,194 & 5,079 \\
\hline & D.P. & 1,001 & 1,036 & 0,895 & 0,888 & 0,910 & 0,930 & 1,058 & 1,066 \\
\hline \multirow{2}{*}{ Homem } & Média & 0,677 & 0,672 & 0,653 & 0,714 & 0,520 & 0,554 & 0,510 & 0,527 \\
\hline & D.P. & 0,468 & 0,470 & 0,476 & 0,452 & 0,500 & 0,497 & 0,500 & 0,499 \\
\hline \multirow{2}{*}{ Branco } & Média & 0,389 & 0,430 & 0,431 & 0,414 & 0,375 & 0,349 & 0,495 & 0,461 \\
\hline & D.P. & 0,488 & 0,495 & 0,495 & 0,493 & 0,484 & 0,477 & 0,500 & 0,498 \\
\hline \multirow{2}{*}{ Urbano } & Média & 0,473 & 0,711 & 0,582 & 0,737 & 0,000 & 0,000 & 1,000 & 1,000 \\
\hline & D.P. & 0,499 & 0,453 & 0,493 & 0,440 & 0,000 & 0,000 & 0,000 & 0,000 \\
\hline \multirow{2}{*}{ Norte } & Média & 0,077 & 0,100 & 0,083 & 0,116 & 0,025 & 0,028 & 0,100 & 0,132 \\
\hline & D.P. & 0,266 & 0,300 & 0,276 & 0,321 & 0,155 & 0,166 & 0,300 & 0,339 \\
\hline \multirow{2}{*}{ Nordeste } & Média & 0,476 & 0,377 & 0,373 & 0,318 & 0,533 & 0,583 & 0,296 & 0,307 \\
\hline & D.P. & 0,499 & 0,485 & 0,484 & 0,466 & 0,499 & 0,493 & 0,457 & 0,461 \\
\hline \multirow{2}{*}{ Sul } & Média & 0,164 & 0,168 & 0,179 & 0,185 & 0,137 & 0,132 & 0,161 & 0,146 \\
\hline & D.P. & 0,370 & 0,374 & 0,384 & 0,389 & 0,343 & 0,339 & 0,368 & 0,354 \\
\hline \multirow{2}{*}{ Centro- Oeste } & Média & 0,096 & 0,097 & 0,122 & 0,101 & 0,089 & 0,085 & 0,111 & 0,111 \\
\hline & D.P. & 0,294 & 0,296 & 0,327 & 0,301 & 0,285 & 0,278 & 0,314 & 0,314 \\
\hline $\begin{array}{l}\text { Número médio de } \\
\text { obs. }\end{array}$ & & 7488 & 17441 & 2818 & 7040 & 15874 & 11124 & 59201 & 59397 \\
\hline
\end{tabular}

